\title{
On the possible observational manifestation of supernova shock impact on the neutron star magnetosphere
}

\author{
A.E. Egorov, K.A.Postnov * \\ Sternberg Astronomical Institute, Universitetski pr. 13, Moscow 119992, Russia
}

\begin{abstract}
Impact of supernova explosion on the neutron star magnetosphere in a massive binary system is considered. The supernova shock striking the NS magnetosphere filled with plasma can lead to the formation of a magnetospheric tail with significant magnetic energy. The magnetic field reconnection in the current sheet formed can convert the magnetic energy stored in the tail into kinetic energy of accelerated charged particles. Plasma instabilities excited by beams of relativistic particles can lead to the formation of a short pulse of coherent radio emission with parameters similar to those of the observed bright extragalactic millisecond radio burst (Lorimer et al. 2007).
\end{abstract}

Subject headings: stars: neutron - radio: bursts - pulsars: general

\section{Introduction}

Supernova (SN) explosion in a binary system with neutron star (NS) is not a rare event. As the binary system can be disrupted during the second SN explosion, the lower limit to the rate of such events can be estimated, for example, from the galactic binary NS formation rate. This rate as inferred from binary pulsar statistics and population synthesis calculations is of order of $10^{-4}-10^{-5}$ per year (Postnov \& Yungelson 2006), i.e. a few - ten events per cubic Gpc per day.

The impact of the SN shock on NS magnetosphere can lead to spectacular observational manifestations. For example, Istomin \& Komberg (2002) argue that it can result in a narrowcollimated jet of hard radiation which can be observed as a gamma-ray burst (GRB). Their arguments are based on the cumulative effect of the SN shock acting on the NS magnetosphere resulting in the formation of a long magnetospheric tail. The magnetic field reconnection in the tail then gives rise to particle acceleration. Synchrotron emission of these particles in the magnetic field can produce hard X-ray photons observed as a gamma-ray burst. Conversion of magnetic energy in the tail into hard X-rays is a complicated physical problem. It is still to be seen if this mechanism can explain the gamma-ray burst phenomenon. However the generation of relativistic particles during magnetic field reconnection seems to be unavoidable (see recent numerical calculations in (Zenitani \& Hoshino 2007)), and a large variety of plasma phenomena are expected to occur during interaction of these particles with the ambient medium.

Here we suggest that plasma instabilities in the process of the magnetic field reconnection can be responsible for the formation of a powerful short non-thermal radio burst. A very short radio burst with peculiar properties was recently discovered by Lorimer et al. (2007). The radio flux of the burst at frequency $1.4 \mathrm{GHz}$ was $30 \pm 10 \mathrm{Jy}$, the duration of the event was

\footnotetext{
*E-mail: astrotula@gmail.com(AEE); kpostnov@gmail.com(KAP)
} 
estimated to be shorter than $5 \mathrm{msec}$ and its dispersion measure was $375 \mathrm{~cm}^{-3}$ pc suggesting the possible extragalactic nature of the source at a distance of $\sim 1 \mathrm{Gpc}$. No host galaxy up to the 18th $B$-magnitude has been found implying a distance limit of $>600 \mathrm{Mpc}$ for a Milky Way-like host. Such a distance implies a radio energy release in the burst of $\sim 10^{40} \mathrm{erg}$ with a brightness temperature of $\sim 10^{34} \mathrm{~K}$ for isotropic radiation. The total rate of such bursts was estimated roughly to be around $90 \mathrm{~d}^{-1} \mathrm{Gpc}^{-3}$, which is much lower than the core-collapse SN rate $\sim 1000 \mathrm{~d}^{-1} \mathrm{Gpc}^{-3}$, but well in excess of the GRB rate $\sim 4 \mathrm{~d}^{-1} \mathrm{Gpc}^{-3}$ and the expected rate of binary neutron star coalescences $\sim 2 \mathrm{~d}^{-1} \mathrm{Gpc}^{-3}$. This estimate, of course, is very uncertain due to a small statistics.

We should note here that properties of the short radio burst reported in Lorimer et al. (2007) are in fact very similar to those of pulsar radio emission: the coherence, an extremely high brightness temperature at frequency $\nu \approx 1.4 \mathrm{GHz}$, a steep spectral shape $\left(\sim \nu^{-4}\right)$. Lorimer et al. (2007) discuss several possible known sources of the strong millisecond radio bursts, including rotating radio transients (RRATs) and giant pulses from radio pulsars, but none of them appear to be energetic enough. Based on statistical arguments only, Popov \& Postnov (2007) proposed that this burst could be produced by a hyperflare of an extragalactic magnetar. Also it was estimated in (Popov \& Postnov 2007) that assuming the ratio of radio emission power to the magneto-rotational losses as observed in giant radio pulses in Crab with a peak flux of $1000 \mathrm{Jy}$, a simple scaling leads to the conclusion that magnetar magnetic fields $\left(B \sim 10^{15} \mathrm{G}\right)$ and millisecond rotation periods are required. However, magnetars are observed to rotate slowly $P=1-10$ s due to rapid spin-down. In the ms magnetar model (Usov 1992, Usov 1994) the characteristic spin-down time is several seconds, so if radio emission were produced in a newly born magnetar by some mechanism, we would observe a series of ms pulses with decreasing period, not a single bright burst.

In contrast, the magnetic field reconnection during relaxation of NS magnetosphere after impact of SN shock naturally leads to the formation of relativistic particle streams. Plasma instabilities in such flows stimulate conversion of kinetic energy of particles to the energy of transverse electromagnetic waves, which can be observed as the short powerfull burst of nonthermal radio emission. Lyubarsky (2008b) has shown that such radio bursts can propagate through the stellar wind without being destroyed by the induced Compton and Raman scattering provided that they originate at distances $>10^{15} \mathrm{~cm}$ from the exploding massive star and their source is moving relativistically. While in massive binary systems such distances from the SN progenitor may be natural, the relativistic bulk motion of the emitting region requires justification. In our model emitting region moves relativistically due to acceleration of electrons during non-stationary magnetic reconnection.

\section{Shock interaction with NS magnetosphere}

Following Istomin \& Komberg (2002), we consider typical SN shock parameters at the free expansion stage after the explosion (Imshennik \& Nadyozhin 1989): the total kinetic energy $E_{\text {kin }} \approx 10^{47} \mathrm{erg}$, the velocity $u \approx 4 \cdot 10^{9} \mathrm{~cm} / \mathrm{s}$, the density $\rho \approx 10^{-8} \mathrm{~g} / \mathrm{cm}^{3}$, and the shock width $h \approx 10^{9} \mathrm{~cm}$.

Assume that the NS was a pulsar just before the explosion, i.e. relativistic plasma was generated near its surface. A young NS can shine as radio pulsar for about $10^{7} \mathrm{yrs}$. In a massive binary, the probability for a SN explosion during this stage is not small, especially if the original binary components had close masses. The characteristic size of the NS magnetosphere (ignoring possible asymmetry due to a strong stellar wind of the secondary component) is about the light cylinder size $R_{l} \sim c / \Omega=c P / 2 \pi$. The NS magnetosphere must be filled with pair plasma with the number density determined by the Goldreich-Julian value (Goldreich \& Julian 1969):

$$
n_{G J}=\frac{\left|\rho_{G J}\right| k}{e}=\frac{|\Omega \mathbf{B}| k}{2 \pi c e},
$$


where $k=10^{4}-10^{5}$ is the particle multiplication factor, $e$ is the elementary charge.

The shock kinetic energy geometrically intercepted by the NS magnetosphere can be estimated as

$$
E^{\prime}=E_{k i n} \frac{\pi R_{l}^{2}}{4 \pi a^{2}}=10^{39}\left(\frac{\Omega}{10 \mathrm{rad} / \mathrm{s}}\right)^{-2}\left(\frac{a}{1 \mathrm{AU}}\right)^{-2} \mathrm{erg},
$$

where $a$ is the distance between the stars. According to Istomin \& Komberg (2002), such shock perturbation can lead to the formation of a magnetospheric tail with substantial magnetic energy. The tail is unstable: magnetic reconnection occurs and charged particles are accelerated to high energies. We suggest that the disruption of the magnetospheric tail can lead to the formation of two colliding relativistic electronic and positronic beams. Plasma instabilities developed in the collision generate plasma waves which further can be converted into transverse electromagnetic waves, giving rise to a narrow-collimated beam of outgoing non-thermal radio emission. The situation here is similar to the most plausible mechanism of radio emission generation in pulsars (Lyubarsky 1992, 2008a).

Istomin \& Komberg (2002) argue that SN shock passing through the NS magnetosphere compresses its head-on part and forms a long narrow magnetospheric tail like in the terrestrial magnetosphere blown by solar wind. In such a magnetospheric tail conditions of the magnetic field freezing into the shock plasma and the magnetic flux conservation in the tail (see Istomin \& Komberg (2002) for more detail) imply that the magnetic field strength in the tail and the tail's diameter at distance $l$ from the NS are

$$
\begin{gathered}
B_{t}=B^{*}\left(\frac{l}{r^{*}}\right)^{1 / 2}, \\
d_{t}=2 r^{*}\left(\frac{l}{r^{*}}\right)^{-1 / 4},
\end{gathered}
$$

respectively. Here

$$
B^{*}=\left(4 \pi \rho u^{2}\right)^{1 / 2} \approx 10^{6}\left(\frac{\rho}{10^{-8} \mathrm{~g} / \mathrm{cm}^{3}}\right)^{1 / 2}\left(\frac{u}{4 \cdot 10^{9} \mathrm{~cm} / \mathrm{s}}\right) \mathrm{G}
$$

is the magnetic field at the Alfvenic radius $r^{*}$ determined as a distance where the magnetic field pressure is balanced by the shock ram pressure. For the dipole magnetic field and the assumed shock parameters we find

$$
r^{*}=R\left(\frac{B}{B^{*}}\right)^{1 / 3}=R\left(\frac{B^{2}}{4 \pi \rho u^{2}}\right)^{1 / 6} \approx 10^{8}\left(\frac{R}{10 \mathrm{~km}}\right)\left(\frac{B}{10^{12} \mathrm{G}}\right)^{1 / 3} \mathrm{~cm}
$$

where $B$ is the field strength at the NS surface, $R$ is the NS radius. So the magnetic energy storage in the narrow $\left(d_{t} \ll L \simeq h\right.$, where $L$ is the tail length), magnetized ( $\left.B^{*} \approx 10^{6} \mathrm{G}\right)$ magnetospheric tail will be

$$
\epsilon_{B}=\int \frac{B_{t}^{2}}{8 \pi} \frac{\pi d_{t}^{2}}{4} d l=\frac{1}{12} B^{* 2} r^{* 3 / 2} h^{3 / 2}=3 \cdot 10^{36}\left(\frac{B^{*}}{10^{6} \mathrm{G}}\right)^{2}\left(\frac{r^{*}}{10^{8} \mathrm{~cm}}\right)^{3 / 2}\left(\frac{h}{10^{9} \mathrm{~cm}}\right)^{3 / 2} \mathrm{erg} .
$$

Clearly, such a tail can be unstable with respect to magnetic reconnection in the current sheet. The symmetry of the problem suggests the plane geometry of the sheet in the orbital plane. During the magnetic reconnection, the electric field component transverse to the tail axis is generated in the plane sheet. This field can accelerate plasma electrons with a broad energy distribution up to high Lorentz factors (see e.g. Zenitani \& Hoshino (2007)). Importantly, some amount of electron-positron plasma is initially expected to be present in the tail with 
the number density below the Goldreich-Julian value (1), since during the formation of the magnetospheric tail electron-positron plasma will outflow along the magnetic lines that have been opened up by the shock. The upper limit on the number density of pair plasma in the tail is

$$
n_{\text {pairs }} \leq n_{G J}(l) \approx 10^{7}\left(\frac{\Omega}{10 \mathrm{rad} / \mathrm{s}}\right)\left(\frac{B}{10^{12} \mathrm{G}}\right)\left(\frac{k}{10^{4}}\right)\left(\frac{l}{5 r^{*}}\right)^{-3} \mathrm{~cm}^{-3} .
$$

At the same time, some amount of electron-ion plasma can penetrate from the shock into the tail during its formation. The number density of electron-ion plasma can significantly exceeds that of pairs.

\section{Generation of radio emission}

At the beginning of the magnetic reconnection, the tail is filled with plasma with admixture of pairs. During the reconnection, several X-points can be formed in the long tail (tearing instability) and a strong electric field directed transversely to the tail axis appears and accelerate charges. Electrons and positrons are accelerated in opposite directions so a low-density positron beam can appear propagating against relativistic electron plasma. The situation becomes similar to the pulsar wind flowing along the open field lines of the NS magnetosphere (Lyubarsky 1992), where tge electric field appears due to leak of Goldreich-Julian charge density (quasistationary violation of condition of full screening of electric field). A fraction of the kinetic energy of accelerated particles can be transformed by plasma instabilities into plasma oscillations mostly at the comoving plasma frequency. These oscillations can then be non-linearly converted into transverse electromagnetic waves observed as pulsar radio emission.

In our case, the duration of the radio pulse produced during magnetic reconnection (fast reconnection with Alfven velocity) can be roughly estimated from the transverse size of the emitting tail: $\tau \approx r^{*} / v_{a} \approx r^{*} / c \approx 3 \mathrm{~ms}$. This time is about as observed in the reported extragalactic radio burst (Lorimer et al. 2007).

The subsequent picture can be as follows. During the magnetic field topology changing, a stream of relativistic electronic plasma moving transversely to the tail axis with some effective mean Lorentz-factor $\Gamma$ can emerge. The reverse flux of positronic plasma accelerated by the same electric field in the reconnecting layer moves with about the same Lorentz-factor against the electrons. Due to various instabilities (beam instability etc.) the kinetic energy of the positronic beam dissipates in the electronic plasma by exciting plasma oscillations. Such energy conversion is effective in the case of small number density of positrons in comparison with electrons $\left(n^{+} \ll n^{-}\right)$, which is expected in our case. These oscillations are non-linearly converted into transversal electromagnetic waves which can be observed as a short radio burst for an appropriate orientation of the tail and the observer. Relativistic aberration confines radio emission within a narrow cone with opening angle $\theta \sim \Gamma^{-1}$. The oscillations occur at the comoving plasma frequency

$$
\tilde{\omega}=\sqrt{\frac{4 \pi e^{2} n^{-}}{m}} .
$$

The conversion rate of plasma oscillations into transversal waves can be expressed as (e.g. Lyubarsky (1992))

$$
\frac{\partial \tilde{W}_{t}}{\partial t}=\tilde{\alpha} \tilde{W}_{t} \tilde{W}_{l}
$$

where

$$
\tilde{\alpha}=\frac{\tilde{\omega}}{n^{-} m c^{2}},
$$

$\tilde{W}_{t}$ and $\tilde{W}_{l}$ are the energy densities of the transversal and longitudinal waves, respectively, in the comoving plasma frame. The observed frequency is Doppler boosted (emitting electrons 
move towards the observer with Lorentz-factor $\Gamma$ ):

$$
\nu \cong 2 \Gamma \tilde{\nu}=2 \Gamma \frac{\tilde{\omega}}{2 \pi}=2 \Gamma e \sqrt{\frac{\tilde{n_{e}^{-}}}{\pi m}},
$$

where $n_{e}^{-}$is the electron number density at the moment of emission. It can be slightly less than the initial number density in the tail since during the magnetic field reconnection the volume of emitting current sheet can widen due to some instabilities (Zenitani \& Hoshino (2007)). We shall adopt $\tilde{n_{e}^{-}}=\alpha n^{-}$, where $\alpha<1$ is the ratio of electron number densities in the current layer and in the tail. The total energy of generated radio emission $E$ can be estimated as the kinetic energy of positrons enhanced by factor $(2 \Gamma)^{2}$. One power of factor $(2 \Gamma)$ appears due to the Doppler effect, and the second power is due to transformation of the kinetic energy of positrons to the frame co-moving with electronic plasma. Below we shall not distinguish between Lorentz-factors of accelerated electrons and positrons. Then

$$
E=\zeta \Gamma N^{+} m c^{2}(2 \Gamma)^{2} \approx 4 \zeta \Gamma^{3} \tilde{n^{+}} \pi r^{* 2} h m c^{2},
$$

where the factor $\zeta<1$ takes into account the efficiency of the kinetic energy conversion into electromagnetic radiation, the efficiency of particle acceleration etc. The $N^{+}$is the total number of positrons, $h$ is the length of the tail. The observed energy flux is then

$$
S=\frac{E}{\tau \Delta_{\nu} \pi(0.5 \theta r)^{2}}=\frac{16 \zeta \Gamma^{5} \tilde{n^{+}} r^{* 2} h m c^{2}}{\tau \Delta_{\nu} r^{2}},
$$

where $\theta=1 / \Gamma$ is the relativistic aberration angle, $\Delta_{\nu}$ is the frequency band of the receiver, $r$ is the distance to the source.

Taking the millisecond radio burst parameters $\nu \approx 1.4 \mathrm{GHz}, S \approx 30 \mathrm{Jy}, r \approx 500 \mathrm{Mpc}$, $\Delta_{\nu} \approx 300 \mathrm{MHz}$ (Lorimer et al. 2007) and assuming $\tilde{n^{+}}=\beta n^{-}, \beta<1, \tau \approx r^{*} / c$ from Eq. (11)-(13) we find

$$
\begin{aligned}
& \Gamma \approx 2000\left(\frac{S}{30 \mathrm{Jy}}\right)^{1 / 3}\left(\frac{h}{10^{9} \mathrm{~cm}}\right)^{-1 / 3}\left(\frac{r}{500 \mathrm{Mpc}}\right)^{2 / 3} . \\
& \left(\frac{r^{*}}{10^{8} \mathrm{~cm}}\right)^{-1 / 3}\left(\frac{\nu}{1.4 \mathrm{GHz}}\right)^{-2 / 3}\left(\frac{\alpha}{0.1}\right)^{1 / 3}\left(\frac{\beta}{0.1}\right)^{-1 / 3}\left(\frac{\zeta}{0.1}\right)^{-1 / 3}, \\
& \tilde{n^{+}} \approx 3 \cdot 10^{3}\left(\frac{\nu}{1.4 \mathrm{GHz}}\right)^{10 / 3}\left(\frac{S}{30 \mathrm{Jy}}\right)^{-2 / 3}\left(\frac{h}{10^{9} \mathrm{~cm}}\right)^{2 / 3} . \\
& \left(\frac{r}{500 \mathrm{Mpc}}\right)^{-4 / 3}\left(\frac{r^{*}}{10^{8} \mathrm{~cm}}\right)^{2 / 3}\left(\frac{\alpha}{0.1}\right)^{-5 / 3}\left(\frac{\beta}{0.1}\right)^{5 / 3}\left(\frac{\zeta}{0.1}\right)^{2 / 3} \mathrm{~cm}^{-3}, \\
& \tilde{n^{-}} \approx 3 \cdot 10^{4}\left(\frac{\nu}{1.4 \mathrm{GHz}}\right)^{10 / 3}\left(\frac{S}{30 \mathrm{Jy}}\right)^{-2 / 3}\left(\frac{h}{10^{9} \mathrm{~cm}}\right)^{2 / 3} . \\
& \left(\frac{r}{500 \mathrm{Mpc}}\right)^{-4 / 3}\left(\frac{r^{*}}{10^{8} \mathrm{~cm}}\right)^{2 / 3}\left(\frac{\alpha}{0.1}\right)^{-5 / 3}\left(\frac{\beta}{0.1}\right)^{2 / 3}\left(\frac{\zeta}{0.1}\right)^{2 / 3} \mathrm{~cm}^{-3}, \\
& E \approx 5 \cdot 10^{32}\left(\frac{S}{30 \mathrm{Jy}}\right)^{1 / 3}\left(\frac{h}{10^{9} \mathrm{~cm}}\right)^{2 / 3}\left(\frac{r}{500 \mathrm{Mpc}}\right)^{2 / 3} . \\
& \left(\frac{r^{*}}{10^{8} \mathrm{~cm}}\right)^{5 / 3}\left(\frac{\alpha}{0.1}\right)^{-2 / 3}\left(\frac{\beta}{0.1}\right)^{2 / 3}\left(\frac{\zeta}{0.1}\right)^{5 / 3} \operatorname{erg} \approx 10^{-4} \epsilon_{B} .
\end{aligned}
$$


The obtained value of the Lorentz-factor does not contradict to constraints imposed by the induced scattering in the source plasma (Lyubarsky 2008b). The positronic plasma number density and the total energy of radio emission accord with Eqs. (7) and (6), respectively.

The spectrum of the observed ms radio burst is $d S / d \nu \sim \nu^{-4 \pm 1}$. As we mention above, the generation mechanism of the burst is analogous to that of pulsar radio emission. Consequently, it is natural that observed spectrum is similar to pulsar radio emission (at the high frequency range, particularly the Crab pulsar has very similar spectrum). Many physical factors can affect the spectral shape: the wide spectrum of plasma waves $(\Delta \tilde{\omega} \sim \tilde{\omega})$, the wide energy spectrum of accelerated particles, plasma inhomogeneities, plasma refraction near the source, extinction in the parent galaxy, etc. The observed spectrum can be partially explained by assuming the power-law energy distribution of accelerated particles $d \tilde{n}^{-} / d \gamma \sim \gamma^{-p}$. The contribution from electrons with Lorentz-factors $[\gamma, \gamma+d \gamma]$ into the observed emission in linear approximation can be written as

$$
d S \sim \gamma d \tilde{n}^{-} \sim \gamma^{1-p} d \gamma .
$$

The additional Lorentz-factor is due to the Doppler effect. Then taking into account (11) and (18) we obtain

$$
\frac{d S}{d \nu}=\frac{d S}{d \gamma} / \frac{d \nu}{d \gamma} \sim \gamma^{1-p} \sim \nu^{1-p}
$$

This spectrum is in agreement with observations provided that $p=4-6$, which generally does not contradict to the energy spectrum of accelerated non-thermal particles found in numerical simulations of relativistic magnetic reconnection (Zenitani \& Hoshino (2007)).

There could be a problem with the generated radio burst passing through the shock. However, the shock can be inhomogeneous and there can be regions with significantly lower densities (holes) transparent for radio emission. The upper limit to the allowed density $\rho^{\prime}$ can be estimated from the condition that the plasma frequency of the shock matter (fully ionized) be less than the frequency of radio waves:

$$
\rho^{\prime} \lesssim m_{p} n_{e}^{\prime}=\frac{\pi m_{p} m_{e} \nu^{2}}{e^{2}} \approx 10^{-14} \mathrm{~g} / \mathrm{cm}^{3} \ll \rho,
$$

where the $n_{e}^{\prime}$ is the electron number density corresponding to the maximum allowed plasma frequency. Indeed, observations of SN1987a suggested strong inhomogeneities in the expanding envelope allowing the early appearance of X-ray emission from the SN remnant (Grebenev \& Syunyaev 1987). The radio emission can also be seen through inhomogeneities, but the observed duration and flux should be modified by effects of passing through such an envelope.

\section{Hard radiation generation}

Let us roughly estimate properties of the possible hard synchrotron emission which can accompany the magnetic reconnection in the magnetospheric tail. The characteristic energy of synchrophotons is

$$
\epsilon_{\gamma}=\hbar \omega_{\gamma} \sim \hbar \frac{e B_{t}}{m c} \Gamma^{2} \approx 40\left(\frac{B_{t}}{10^{6} \mathrm{G}}\right)\left(\frac{\Gamma}{2000}\right)^{2} \mathrm{keV},
$$

which falls into the soft gamma-ray energy range. The upper limits to the total energy of this radiation $E_{\gamma}$ and the flux $S_{\gamma}$ can be estimated from Eqs. (3), (14), (16) with account for the synchrotron intensity from one particle $I=(2 / 3)\left(e^{4} B_{t}^{2}\right) /\left(m^{2} c^{3}\right) \Gamma^{2}$ :

$$
\begin{aligned}
E_{\gamma}=I N^{-}=\frac{2 e^{4} B_{t}^{2} \Gamma^{2}}{3 m^{2} c^{3}} n^{-} \pi r^{* 2} h \approx & 4 \cdot 10^{30}\left(\frac{B_{t}}{10^{6} \mathrm{G}}\right)^{2}\left(\frac{\Gamma}{2000}\right)^{2} \\
& \left(\frac{\tilde{n^{-}}}{3 \cdot 10^{4} \mathrm{~cm}^{-3}}\right)\left(\frac{r^{*}}{10^{8} \mathrm{~cm}}\right)^{2}\left(\frac{h}{10^{9} \mathrm{~cm}}\right)\left(\frac{\tau}{3 \mathrm{~ms}}\right) \mathrm{erg},
\end{aligned}
$$




$$
\begin{aligned}
S_{\gamma}=\frac{E_{\gamma}}{\pi(0.5 \theta r)^{2}} \approx 10^{-14} & \left(\frac{B_{t}}{10^{6} \mathrm{G}}\right)^{2}\left(\frac{\Gamma}{2000}\right)^{4} . \\
& \left(\frac{\tilde{n^{-}}}{3 \cdot 10^{4} \mathrm{~cm}^{-3}}\right)\left(\frac{r^{*}}{10^{8} \mathrm{~cm}}\right)^{2}\left(\frac{h}{10^{9} \mathrm{~cm}}\right)\left(\frac{r}{500 \mathrm{Mpc}}\right)^{-2} \frac{\mathrm{erg}}{\mathrm{s} \cdot \mathrm{cm}^{2}} .
\end{aligned}
$$

Apparently, the total energy of hard non-thermal radiation is similar to that of radio emission (17), but the flux falls short of the sensitivity of modern detectors. So no accompanying "gamma-ray burst" is expected, which agrees with the absence of any gamma-ray event at the time of the observed radio burst (Lorimer et al. 2007). Clearly, should this happen in our Galaxy or in the Local Group, both radio and gamma-ray bursts can be observed, probably associated with an emerging supernova.

\section{Discussion}

We have shown that a bright millisecond burst of non-thermal radio emission with properties similar to those observed by Lorimer et al. (2007) can be explained by the supernova shock interaction with NS magnetosphere in a massive binary system. The shock passing through the magnetosphere leads to the formation of a long magnetospheric tail (Istomin \& Komberg 2002). We conjecture that the magnetic energy stored in the tail can be transformed into the kinetic energy of non-thermal particles due to magnetic reconnection. If there is some fraction of positrons (which seems reasonable if NS before the SN explosion was at the pulsar stage), plasma instabilities can transform the kinetic energy of the less dense positronic beam into the plasma oscillations in the electron beam which can then be non-linearly converted into transversal electromagnetic waves, like in pulsar magnetospheres (Lyubarsky 1992), thereby producing a short pulse of non-thermal radio emission. The magnetic fields of young NSs can vary in a wide range from $10^{11}$ to $10^{15} \mathrm{G}$, leading to a broad range of magnetospheric sizes, densities of pair plasma filling the magnetosphere, etc. The situation is even more diverse in massive binary systems with strong stellar winds from presupernova stars. In addition, the density and velocity of SN shocks can also vary significantly. So all basic parameters of our model $\left(r^{*}, \tilde{n^{+}}, \tau, \Gamma\right.$ and $\tilde{n^{-}}$) can be very different, and so should be the properties (frequency, flux, duration) of possible radio transients accompanying the magnetosphere topology restructuring during reconnection.

Magnetic reconnection can also occur in the region with toroidal magnetic field beyond the light cylinder of a pulsar. In this case the current sheet is located in the rotational equatorial plane of the pulsar. The magnetic field strength at the light cylinder $B_{l}$ can be significant:

$$
B_{l} \approx B\left(\frac{R}{R_{l}}\right)^{3} \approx 4 \cdot 10^{5}\left(\frac{B}{10^{13} \mathrm{G}}\right)\left(\frac{\Omega}{100 \mathrm{rad} / \mathrm{s}}\right)^{3} \mathrm{G} .
$$

Hence there is a substantial magnetic energy storage $\epsilon_{B} \sim\left(B_{l}^{2} / 8 \pi\right) R_{l}^{3} \sim 10^{35}$ erg outside the magnetosphere (taking into account that toroidal magnetic field decreases slowly as $1 / r$ ). A stationary reconnection of the toroidal field was suggested by Lyubarsky (1996) to explain hard emission of pulsars. In our scenario, the passing shock compresses the NS magnetosphere and can initiate a non-stationary large-scale reconnection of the toroidal magnetic field beyond the light cylinder. Estimates similar to those given in Section 3 can explain the generation of short radio burst in two-dimensional current sheet approximation. Its properties can be similar to those observed by Lorimer et al. (2007) assuming model parameters similar to those given by Eqs. (14)-(17) (provided that positrons, which excite plasma waves, are collected from a large volume $\sim R_{l}^{3}$ ). Such parameters do not contradict all existing constraints, so this approach can be considered as an alternative model for the millisecond radio burst. In this case it is not needed to explain the transparency of the shock to the emergent radio emission since the radio pulse can be produced before the SN shock has passed the magnetosphere and screened the source. 
The search for other short radio transients is under way (Lorimer et al. 2007), and larger statistics can be used in future to test the proposed model.

\section{Acknowledgements}

The authors thank Profs. B.V. Somov and V.V. Kocharovsky for useful discussions. A valuable feedback from Dr. Yu. Lyubarsky is also acknowledged. The work is partially supported by the RFBR grant 07-02-00961.

\section{References}

Goldreich P., Julian W.H., 1969, ApJ, 157, 869

Grebenev S.A., Syunyaev R.A., 1987, Pis'ma Astron. Zh., 13, 1024

Imshennik V.S., Nadyozhin D.K., 1989, Sov. Sci. Rev., Sect. E, Vol. 8, Part 1, p. 1

Istomin Ya.N., Komberg B.V., 2002, Astron. Rep., 46, 908

Lorimer D.R., Bailes M., McLaughlin M.A., Narkevic D.J., Crawford F., 2007, Science, 318,777

Lyubarskii Yu.E., 1992, AA, 265, L33

Lyubarskii Y.E., 1996, AA, 311, 172

Lyubarsky Y.E., 2008a, In 40 Years of Pulsars. AIP Conf. Proc. 983, p. 29

Lyubarsky Yu., 2008b, arXiv:0804.2069

Popov S.B., Postnov K.A., 2007, arXiv:0710.2006

Postnov K.A., Yungelson L.R., 2006, LRR, 6

Usov V., 1992, Nature, 357, 472

Usov V., 1994, MNRAS, 267, 1035

Zenitani S., Hoshino M., 2007, arXiv:0708.1000 